\title{
Left Adrenal Tumor Disappeared Outside a Field of Vision During Laparoscopic Surgery: The True Identity Was Gastrointestinal Stromal Tumor
}

\author{
Hiroshi Ushida, MD, PhD, ${ }^{1,2}$ Hirofumi Goto, MD, Satoshi Ishitoya, MD, PhD, ${ }^{2,3}$ and Hiroyuki Onishi, MD, PhD ${ }^{2,4}$
}

\begin{abstract}
Background: We report a case of gastrointestinal stromal tumor (GIST) mimicking a left adrenal tumor.

Case Presentation: A 62-year-old female was referred to our hospital for the treatment of left adrenal tumor of $2.8 \times 2.3 \mathrm{~cm}$ incidentally found during her annual checkup. The preoperative diagnosis based on upper gastroscopy and imaging complete examinations was nonfunctional left adrenal tumor possessing malignant potential. Transperitoneal laparoscopic left adrenalectomy was performed. However, the tumor was not found in the excised adrenal gland that had been completely removed during surgery. Repeat computed tomography revealed the presence of the same tumor. Finally, reoperation led us to the true diagnosis of GIST.

Conclusion: Many urologists are not familiar with GIST. It is necessary to take GIST into consideration when left adrenal tumor close to the stomach is diagnosed.

We discuss the traps which we fell into during the perioperative period in this case.
\end{abstract}

Keywords: gastrointestinal stromal tumor, laparoscopic surgery, left adrenal tumor, preoperative diagnosis, snake retractor

\section{Introduction and Background}

G ASTROINTESTINAL STROMAL TUMOR (GIST) is the most common mesenchymal tumor of the gastrointestinal (GI) tract, and accounts for $0.1 \%$ to $3.0 \%$ of all GI malignancies. ${ }^{1}$ On the other hand, GIST is rarely seen near the urinary tract. In a literature review, a few cases of GIST presenting as a left adrenal tumor were reported. ${ }^{2,3}$ Most adrenal tumors are diagnosed by imaging studies, which are important modalities in preoperative diagnosis. This preoperative information generally leads us to performing the correct treatment. However, in our case, we were entrapped by preoperative misdiagnosis. Moreover, we retrospectively deduced that the snake retractor, which was used to develop the operative field for laparoscopic surgery, pushed the true tumor away outside the field of vision. Herein, we report such a case and detail the traps which we fell into.

\section{Presentation of Case}

A 62-year-old female was referred to our hospital for the treatment of left adrenal tumor, which had been incidentally found during complete examinations for a gastric submucosal protruding mass in her annual gastroscopic examination. She did not suffer from digestive symptoms. Abdominal CT showed a soft-tissue density mass of $2.8 \times 2.3 \mathrm{~cm}$ in maximum diameter without intravenous contrast enhancement on top of the left kidney (Fig. 1A). Magnetic resonance imaging revealed a mass with a diameter of about $3 \mathrm{~cm}$ close to the top of the left kidney. Positron emission tomography-CT showed low-signal accumulation in the tumor, which implied malignant potential (Fig. 1B). Endocrine examination showed normal adrenal hormonal profiles. The preoperative diagnosis was a nonfunctional left adrenal tumor with malignant potential. Transperitoneal laparoscopic left adrenalectomy

${ }^{1}$ Department of Urology, Japan Community Health Care Organization Shiga Hospital, Otsu, Japan.

${ }^{2}$ Department of Urology, Shiga Medical Center for Adults, Moriyama, Japan.

${ }^{3}$ Department of Urology, Otsu Red Cross Hospital, Otsu, Japan.

${ }^{4}$ Department of Urology, Osaka Red Cross Hospital, Osaka, Japan.

(C) Hiroshi Ushida et al. 2017; Published by Mary Ann Liebert, Inc. This is an Open Access article distributed under the terms of the Creative Commons Attribution License, which permits unrestricted use, distribution, and reproduction in any medium, provided the original work is properly cited. 

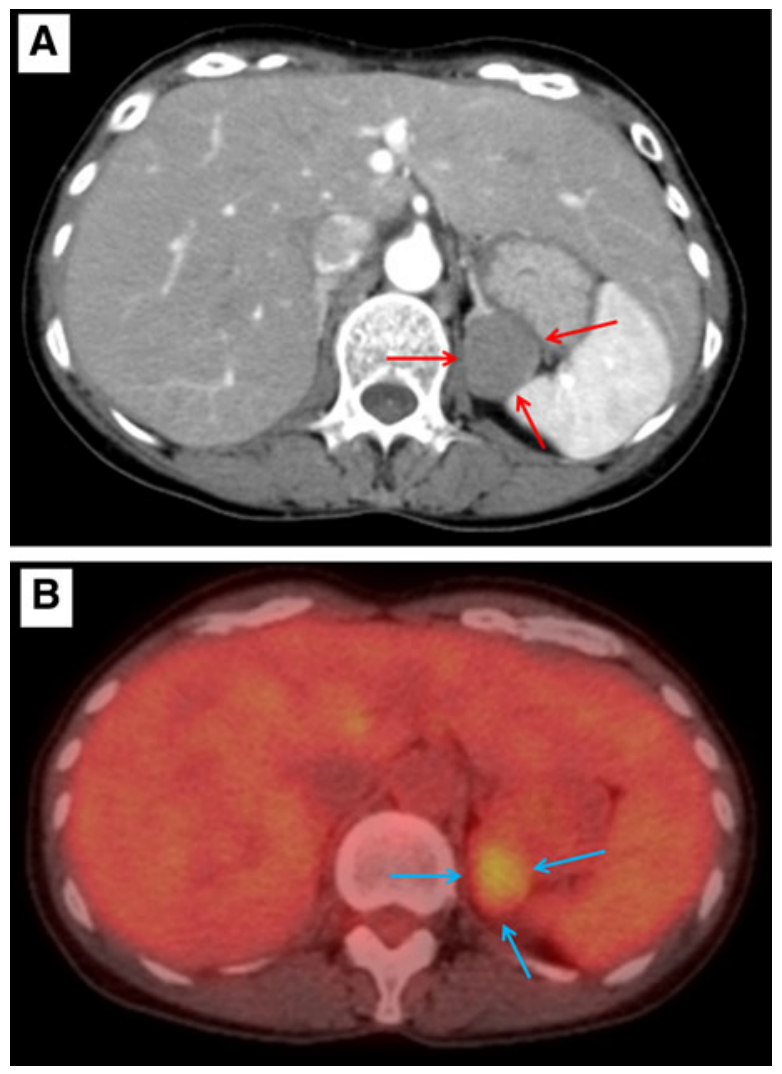

FIG. 1. (A) Enhanced abdominal CT. A soft-tissue density mass (arrows) of smaller than $3 \mathrm{~cm}$ is detected adjacent to a normal left adrenal gland. (B) Positron emission tomographyCT. Low-signal accumulation being consistent with the tumor (arrows) implies malignant potential.

was performed. During the surgery, we believed that the tumor was hiding in the adipose tissue of Gerota's fascia on top of the left kidney (Fig. 2). We completed the laparoscopic left adrenalectomy as planned. The extracted specimen was intactly submitted for pathological examination.

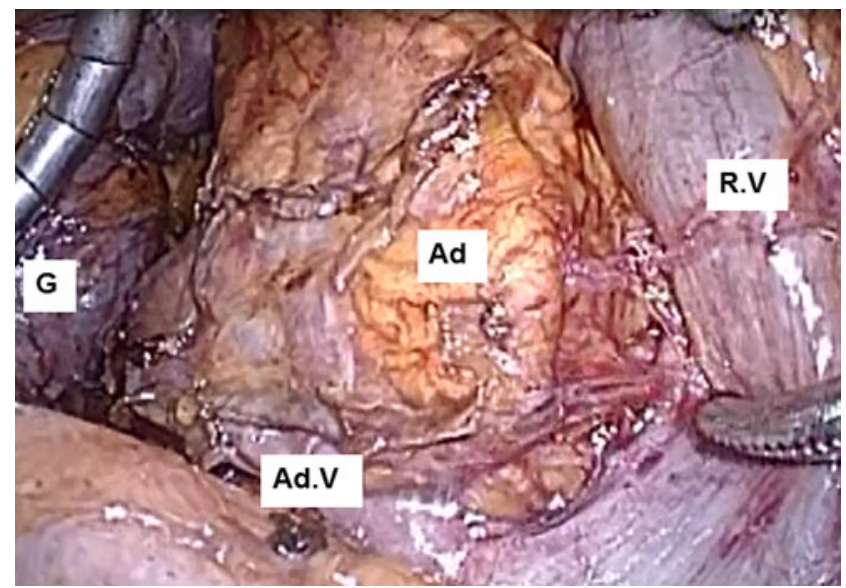

FIG. 2. Intraoperative view during laparoscopic left adrenalectomy. The snake retractor pushes the tumor away outside a field of vision with the spleen. Ad, adrenal gland; Ad.V, adrenal vein; G, GIST; GIST, gastrointestinal stromal tumor; R.V, renal vein.

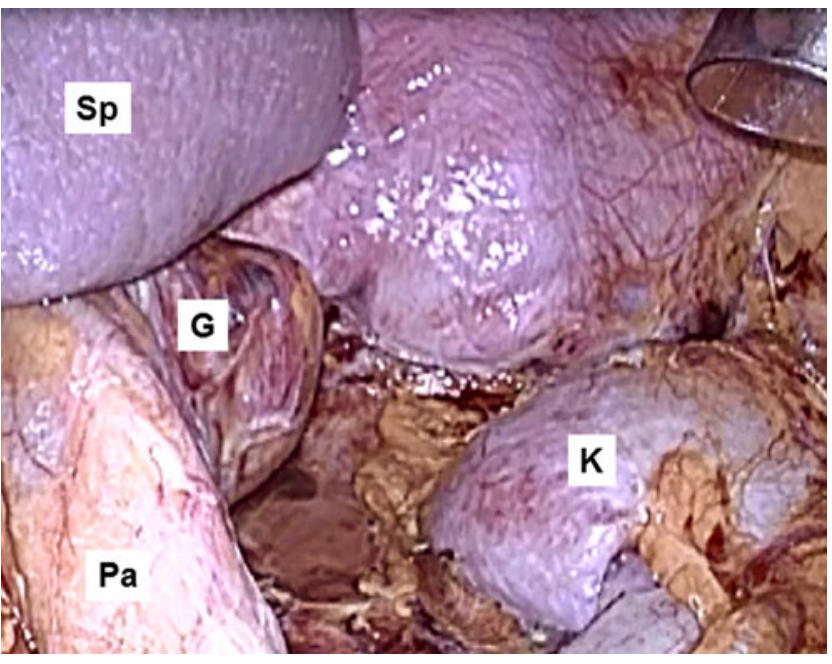

FIG. 3. Intraoperative view during the laparoscopic reoperation. No residual tissue is seen on top of the left kidney. GIST is found over the peritoneum. G, GIST; K, kidney; Pa, pancreas; Sp, spleen.

Pathological examination revealed that there was no tumor in the specimen. Emergency CT scan, which was performed to confirm the existence of the tumor, showed the same tumor close to the top of the left kidney. We performed laparoscopic reoperation by the same approach on that day. First, we confirmed that there was no residual tissue on top of the left kidney; however, the tumor was found over the peritoneum (Fig. 3). The tumor existed between the greater curvature of the stomach and the splenic hilum (Fig. 4). The tumor originated from the greater curvature of the stomach, and the tumor was suspected of being a GIST. Finally, partial gastrectomy was performed by laparotomy. Pathological examination showed a $2.8 \mathrm{~cm}$, whitish, elastic tumor beneath the gastric smooth muscle. The pathological diagnosis was spindle cell-type GIST. The mitotic count was $<5$ mitoses per 50 highpower fields. Tumor cells were immunohistochemically positive for KIT and CD34, and negative for smooth muscle actin

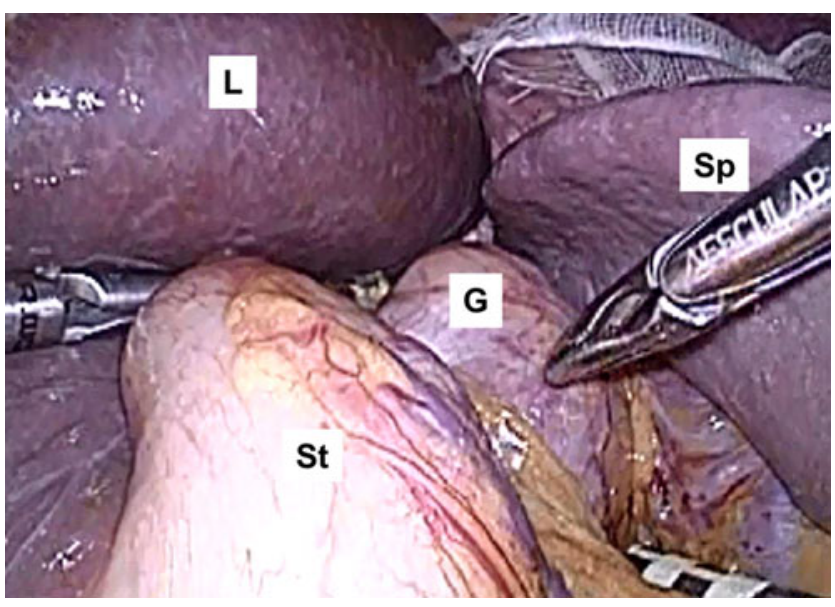

FIG. 4. Intraoperative view during the laparoscopic reoperation. A GIST exists between the greater curvature of the stomach and the splenic hilum. G, GIST; K, kidney; L, liver; Sp, spleen; St, stomach. 
(SMA) and S-100 protein. Hence, the diagnosis of GIST was confirmed. No adjuvant therapy with imatinib was prescribed. Postoperative follow-up CT during surveillance revealed no local tumor recurrence and metastasis 36 months after the partial gastrectomy.

\section{Discussion and Literature Review}

There were several traps during the perioperative period that led to the wrong diagnosis and treatment in the present case. We describe each as follows.

\section{Preoperative trap}

GISTs represent the most common mesenchymal neoplasm of the GI tract. With an annual incidence of 11 to 14 per $10^{6}$, they form $0.1 \%$ to $3.0 \%$ of GI malignant tumors. ${ }^{1}$ GISTs have been documented in all parts of the GI tract; however, they are rarely seen near the urinary tract. Seventy percent of patients with GIST are symptomatic, whereas $20 \%$ are asymptomatic and detected incidentally. ${ }^{1}$ It is difficult to make a definitive diagnosis of a small gastric submucosal mass, even by upper GI examination and imaging studies. In our case, they suggested a left adrenal tumor rather than a gastric submucosal tumor. In this way, we were led to the misdiagnosis of left adrenal tumor. This was the first pitfall.

\section{Intraoperative trap}

We performed the laparoscopic operation under the misdiagnosis of left adrenal tumor. We believed that the tumor was hiding in the adipose tissue of Gerota's fascia on top of the left kidney. In addition, we retrospectively deduced that the snake retractor pushed the tumor outside the field of vision along with other abdominal organs (spleen, pancreas, and stomach) (Fig. 2). This was the second pitfall. We performed left adrenalectomy without excising the true tumor.

\section{Postoperative trap}

The excised specimen was submitted for pathological examination without checking the existence of the tumor. This was the third pitfall.

In this way, we were caught in some traps. A small GIST of less than $3 \mathrm{~cm}$ in our case contributed to the misdiagnosis, although the median size of GISTs is generally between 5 and $8 \mathrm{~cm} .{ }^{1}$ In case of a small GIST, it is difficult to reach the correct diagnosis preoperatively with upper GI endoscopy and imaging studies. Consequently, the misdiagnosis led to the wrong treatment. Furthermore, a snake retractor, which had been used to develop the operative field for laparoscopic surgery, moved the tumor outside the field of vision (Fig. 2). Our only regret was not to have confirmed the specimen.

When we suspected of being a GIST during the surgery, we had to consult a digestive surgeon. Surgical resection is the mainstay of treatment for localized gastric GIST. It is imperative to avoid tumor rupture. The tumors are fragile and should be handled with care, with an aim to achieve complete gross resection of the tumor with an intact pseudocapsule.

It is important to perform immunohistochemical staining to make a definitive diagnosis. The majority of GISTs (93.6\%) are positive for KIT (CD117). In $80.1 \%$ of cases, immunohistochemistry for CD34 is also positive. SMA and S-100 protein are positive in $28.2 \%$ and $20.2 \%$ of cases. ${ }^{4}$
GISTs contain activated c-KIT mutations, which play a central role in the pathogenesis of GIST, and mutations of cKIT resulted in gain of function of the enzymatic activity of KIT tyrosine kinase. Imatinib, which is a molecular target drug for KIT, has a very high likelihood of clinical benefit. A survival benefit by imatinib was seen in patients with a high risk of recurrence (mitotic count $>5 / 50$ high power field, size $>5 \mathrm{~cm}$, nongastric location, and tumor rupture). ${ }^{1}$ Our patient did not meet the criteria for adjuvant therapy with imatinib, and this therapy was not administered.

\section{Conclusion}

We experienced a case of GIST mimicking left adrenal tumor, which led us to the wrong treatment by three steps during the perioperative period.

Many urologists are not familiar with GIST. It is necessary to take GIST into consideration when left adrenal tumor close to the stomach is diagnosed.

\section{Disclosure Statement}

No competing financial interests exist.

\section{References}

1. Rammohan A, Sathyanesan J, Rajendran K, et al. A gist of gastrointestinal stromal tumors: A review. World J Gastrointest Oncol 2013;5:102-112.

2. Chung SD, Chueh JS, Yu HJ. Laparoscopic resection of gastric gastrointestinal stromal tumors presenting as left adrenal tumors. World J Gastroenterol 2012;18:96-98.

3. Hasebe K, Satoh M, Komori K, et al. [Gastrointestinal stromal tumor of the stomach mimicking adrenal tumor: A case report] (Jpn). Hinyokika Kiyo 2004;50:853-855.

4. Kim KM, Kang DW, Moon WS, et al. Gastrointestinal stromal tumors in Koreans: It's incidence and the clinical, pathologic and immunohistochemical findings. J Korean Med Sci 2005;20:977-984.

Address correspondence to:
Hiroshi Ushida, MD,PhD
Department of Urology
Japan Community Health Care Organization
Shiga Hospital
16-1, Fujimidai
Otsu 520-0846
Shiga
Japan
E-mail: ushidah@za.ztv.ne.jp

$\begin{aligned} & \text { Abbreviations Used } \\ \text { CT } & =\text { computed tomography } \\ \text { GI } & =\text { gastrointestinal } \\ \text { GIST } & =\text { gastrointestinal stromal tumor } \\ \text { SMA } & =\text { smooth muscle actin }\end{aligned}$

Cite this article as: Ushida $\mathrm{H}$, Goto $\mathrm{H}$, Ishitoya $\mathrm{S}$, Onishi H (2017) Left adrenal tumor disappeared outside a field of vision during laparoscopic surgery: the true identity was gastrointestinal stromal tumor, Journal of Endourology Case Reports 3:1, 102-104, DOI: 10.1089/cren.2017.0065. 